\title{
Effects of smartphone mirroring-based telepresence exercise on body composition and physical function in obese older women
}

\author{
Jeeyoung Hong ${ }^{1,2} \cdot$ Suk Wha Kim ${ }^{3} \cdot$ Hyunjin Joo ${ }^{3,4} \cdot$ Hyoun-Joong Kong ${ }^{3,4,5,6}$
}

Received: 12 August 2021 / Accepted: 15 November 2021 / Published online: 14 January 2022

(c) The Author(s), under exclusive licence to Springer Nature Switzerland AG 2021

\begin{abstract}
Aims This study aimed to develop a smartphone mirroring-based telepresence exercise program that can be performed at home while allowing for real-time feedback by instructors.

Methods For this randomized controlled trial, 29 obese older women aged 66-87 years with $\geq 30 \%$ body fat were recruited at a senior citizen center. The intervention group was provided with the smartphone mirroring-based telepresence exercise program, in which participants exercised in their homes for 20-40 min three times a week for 12 weeks. Participants in the control group performed the same exercise program at the senior citizen center. Body composition and functional abilities were measured before and after the program.

Results Women in the intervention group showed a decrease in their body fat percentage $(P=0.026)$ and an increase in grip strength $(P=0.008)$. In the control group, women demonstrated a decrease in their weight $(P=0.006)$ and body fat percentage $(P=0.001)$ and an increase in skeletal muscle $(P=0.044)$ and grip strength $(P=0.006)$.

Conclusion Smartphone mirroring-based telepresence exercises at home lower body fat percentage and increase muscle strength similar to traditional group exercises. They present an innovative way for obese older women to improve and maintain their health.

Trial registration Clinical Research Information Service KCT0006147.
\end{abstract}

Keywords Aging $\cdot$ Body composition · Physical function · Obesity $\cdot$ Smartphone

\section{Introduction}

Hyoun-Joong Kong

69005@ snuh.org; gongcop7@snu.ac.kr;

gongcop@gmail.com

1 Department of Life Sports Educators, Kongju National University, Kongju, Republic of Korea

2 Institute of Medical and Biological Engineering, Medical Research Center, Seoul National University College of Medicine, Seoul, Republic of Korea

3 Medical Big Data Research Center, Seoul National University College of Medicine, Seoul, Republic of Korea

4 Transdisciplinary Department of Medicine and Advanced Technology, Seoul National University Hospital, 101 Daehak-ro, Jongno-gu, Seoul 03080, Republic of Korea

5 Department of Biomedical Engineering, Seoul National University College of Medicine, Seoul, Republic of Korea

6 AI Institute, Seoul National University, Seoul, Republic of Korea
In 2019 , the number of people aged $\geq 65$ years in South Korea was $7,685,000$, making up $14.9 \%$ of the country's rapidly aging population. The life expectancy of South Koreans increased from 76.01 years in 2000 to 82.36 years in 2016, which has led to heightened interest in health care for senior citizens [1].

Among the changes in body composition that occur with aging, the decrease in muscle mass and increase in body fat percentage are particularly problematic [2]. They may result in obesity and cardiovascular disease because of decreased muscle function and insulin resistance [3].

Obesity is considered a multi-system chronic recurrent disease process that adversely affects almost all physiological functions of the body and increases morbidity and mortality [4]. It is associated with many metabolic disorders and comorbidities that interfere with quality of life and work productivity and increase medical costs [5]. In particular, it is believed that after menopause, the secretion of follicle 
hormones and prevention of the activation of lipoprotein lipases decrease, increasing the rate of obesity and threatening the health of old aged women [6]. Obese women have low body function [7] and the average body fat rate after the age of 70 is $39 \%$, which is reported to be higher than that of elderly males (21\%) [8].

There are various ways to solve this problem. One of the most effective methods is exercise, which has been shown to slow the pace and positively affect the pattern of aging [9]. In South Korea, various exercise programs are provided to the older people to reduce their body fat and increase their muscle mass. Most of these are delivered as group activities in indoor spaces, such as senior citizen's centers, welfare centers, village halls, and gymnasiums [10, 11].

However, if the location of the exercise class is far from the home of the elderly, those at risk for falls are restricted from participating; moreover, attendance, in general, depends on the season and weather [12]. Furthermore, largescale epidemics or pandemics, such as the coronavirus disease 2019 (COVID-19) pandemic, and increasing environmental concentrations of micro- and ultra-fine dust are also restricting older people's opportunities to exercise outdoors.

According to a recent study, during the COVID-19 pandemic, the physical activity duration of adults decreased by $60 \%$, while the sedentary duration increased by $42 \%$ [13]. In addition, social distancing has reduced all additionally planned physical activities, especially harmful to the elderly [14]. Therefore, active efforts by experts are needed to maintain the physical activity and exercise levels of the elderly during the COVID-19 pandemic.

As information technology continues to develop and becomes more user-friendly, operating a smartphone is becoming easier for everyone, including the older people. The rapid and widespread uptake of the use of smartphones over the past 10 years has led to the introduction and development of mobile health, and the effectiveness of various health behavior interventions delivered in this way has been investigated in previous studies $[15,16]$. Smartphone-based interventions have advantages over traditional face-to-face consultations in that they can provide individualized health information and management more frequently and continuously [17].

In 2018 , the internet usage rate of the older people was reported to be at $88.8 \%$ for those in their $60 \mathrm{~s}$ and $38.6 \%$ for those aged $70 \mathrm{~s}$ and older [1]. However, current smartphone app-based exercise programs are unsuited to effectively provide feedback tailored to the needs of older people, who may have impaired cognitive abilities. These challenges can be overcome if exercise instructors give older people real-time feedback that is easily comprehensible.

Therefore, this study aimed to implement a mirroringbased telepresence exercise program that is provided on smartphones, can be safely performed at home, and enables real-time feedback from the exercise instructors to the participating obese older women. Furthermore, the effects of the program on the body composition and physical function of the participants were investigated.

\section{Materials and methods}

\section{Participants}

Thirty-one obese ( $>30 \%$ body fat) older ( $>65$ years) women were recruited from a senior citizen center in Gangseo-gu, Seoul, South Korea. Recruitment was performed in person by a research coordinator. The women were assigned to either the intervention group (IG, $n=12$ ) or the control group (CG, $n=19)$ using random block sampling. Of the 31 women enrolled, two discontinued the intervention for medical reasons and did not undergo the post-test assessment. The remaining 29 participants completed the study, including the pre- and post-test assessments, and their data were included in the analysis (Fig. 1).

All procedures of the study have been conducted according to the principles expressed in the Declaration of Helsinki 1964 and its later amendments. This study was approved by the Seoul National University Hospital Institutional Review Board (approval no. 1804-060-936) and was registered with the Clinical Research Information Service, which is a primary registry of the World Health Organization International Clinical Trials Registry Platform. All participants provided written informed consent before enrollment and were free to withdraw from the study at any time.

\section{Intervention group}

\section{Equipment}

This study was designed as a double-blind, parallel-group, randomized controlled trial.

This study used a mixed design, with measurement time and group as independent variables and body composition and physical function as dependent variables. The dependent variables were measured in a pre-test 1 week before the application of the exercise program.

Participants and the exercise instructor were each provided with a smartphone (Galaxy 4 note, Samsung Group, Seoul, South Korea), 24-inch LCD display monitor (Newsync X24 Real 144 Sonic Boom, Bitm), a smartphone mirroring device (Miracast MRC-01, Actto), a folding chair, resistance bands (TheraBand ${ }^{\circledR}$; The Hygenic Corporation, Akron, OH, USA), and an exercise mat. 
Fig. 1 Flow diagram of the study design

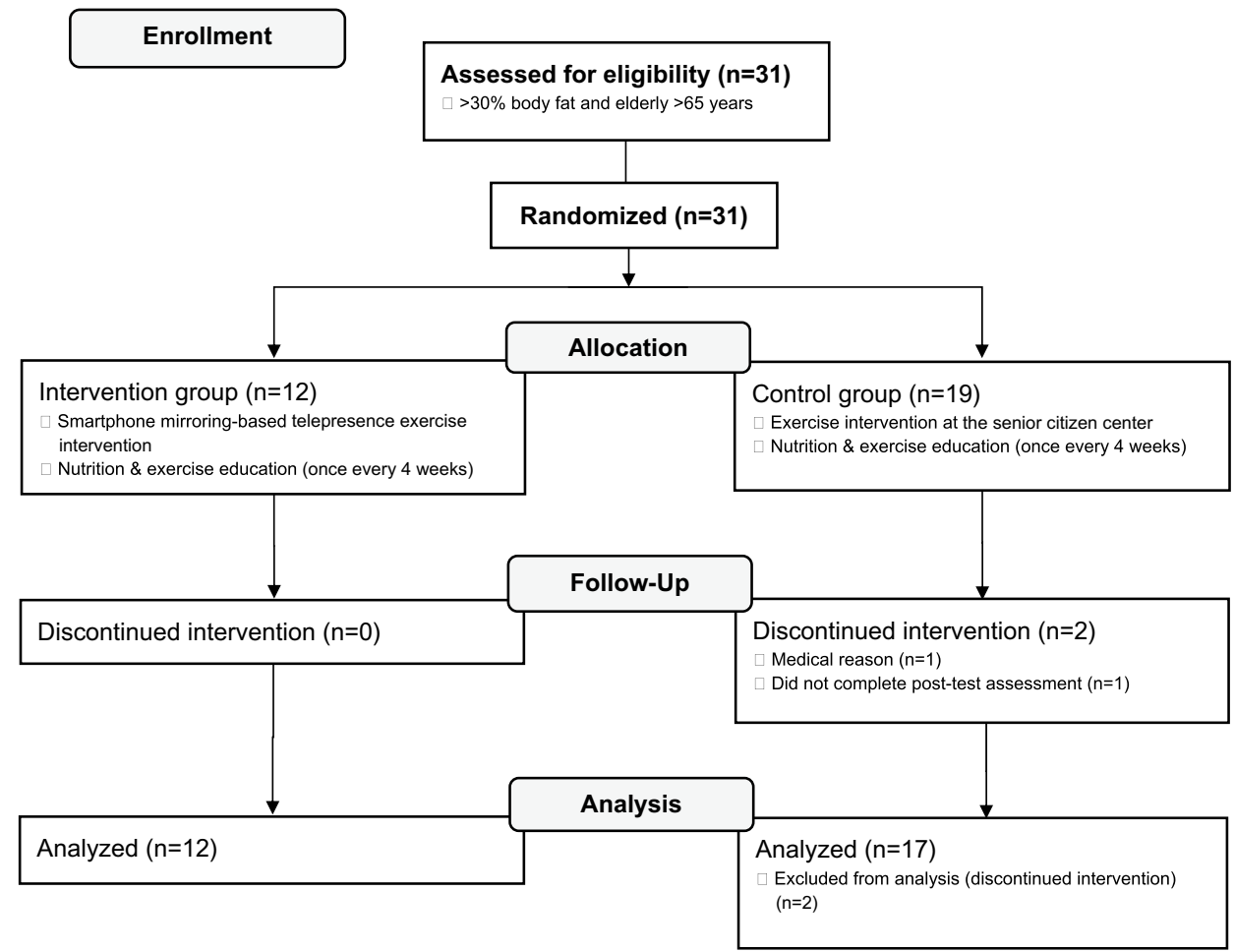

\section{Smartphone mirroring-based telepresence exercise program}

The smartphone mirroring-based telepresence platform was developed using Node.js ${ }^{\circledR}$ (Open JS Foundation, https:// nodejs.org/en/), PHP programing language (The PHP Group, https://www.php.net/downloads), and the MySQL database (Oracle, Austin, TX, USA) on a Windows Server 2008 operating system (Microsoft Corporation, Redmond, WA, USA). A web real-time communication module that conducts video conversations with subjects was developed using Node.js ${ }^{\circledR}$. Modules regarding participants' membership, login, usage history management, and session rooms were developed in PHP and MySQL [18].

All sessions were performed on a one-on-one basis based on a predefined schedule. The exercise instructor opened a classroom by accessing the program web address (https:// healthcare8000.iptime.org) using a smartphone at the designated time and waited until the participant connected. The website was accessed via the web browser (Google Chrome v. 66.0.3359.139, Google, Mountain View, CA, USA) installed on the smartphone.

Participants accessed the web address at the scheduled time to meet with the exercise instructor in the classroom. The internet connection used in this study was at most $100 \mathrm{Mbps}$ fiber optic LAN, and all smartphones were connected through their wireless routers. Although a difference was found between the internet speeds at the participants' residential environment and the location of the router, the study was conducted in an environment with an internet speed of at least $20 \mathrm{Mbps}$.

The screen and sound of the smartphone were transmitted to the large screen display using Miracast technology. Remote access software (TeamViewer QuickSupport 13.0.7847 https://play.google.com/store/apps/details?id= com.teamviewer.quicksupport.market) was installed on the participants' smartphones to provide them with technical support, if needed.

The exercise instructor who had a major in exercise physiology, connected the wireless headset to the smartphone and conducted the exercise program in her personal studio. The overall architecture of the smartphone mirroring-based telepresence exercise platform is shown in Fig. 2.

The smartphone telepresence exercise program was based on the guidelines of the American College of Sports Medicine [19] and exercise manual for obesity management published by the Korean Society for the Study of Obesity [20]. Exercise intensity was controlled using the rate of perceived exertion measured with the Borg scale [21] and progressively increased from RPE 11 to RPE 15 [22]. The total exercise duration was progressively increased by $20-40 \mathrm{~min}$ during the intervention period. The RPE and total exercise duration were gradually increased every 4 weeks.

The main exercise activity comprised resistance exercises performed using color-coded resistance bands and a chair. Details of the telepresence exercise programs are shown in Table 1 and Fig. 3. 


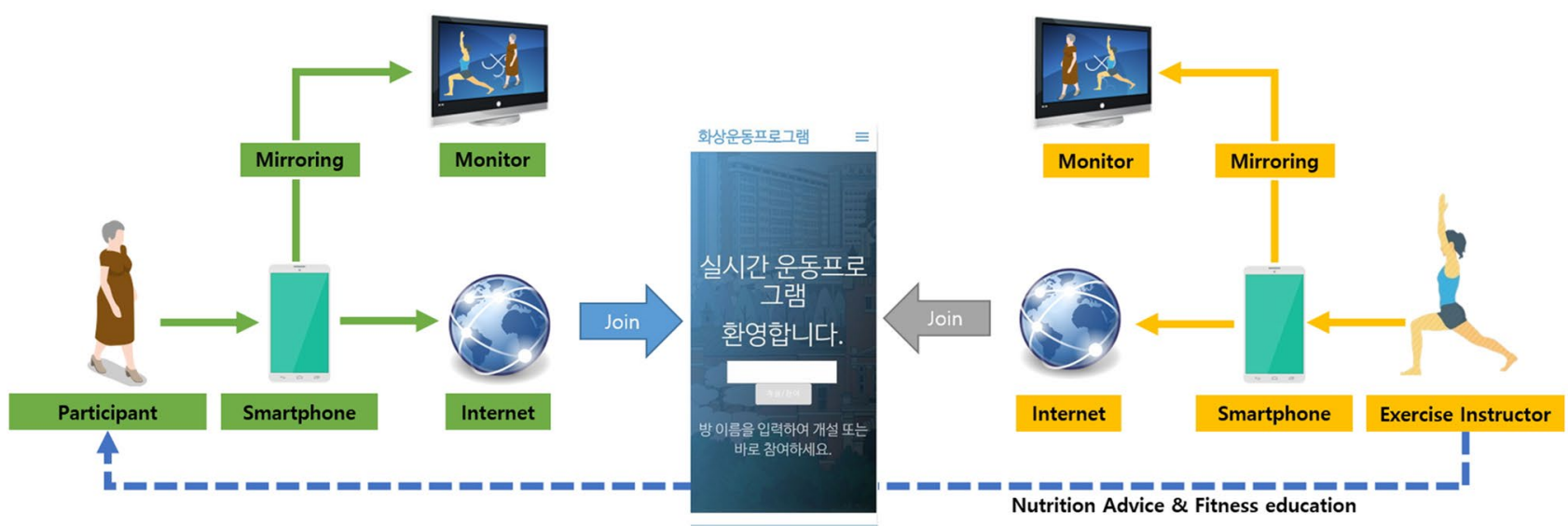

Fig. 2 Overview of the telepresence exercise platform using web real-time communication technology developed for this study in obese older women

Table 1 Exercise program design for the intervention and control groups in obese older women $(n=29)$

\begin{tabular}{|c|c|c|c|c|}
\hline \multirow{2}{*}{$\frac{\text { Program part }}{\text { Warm-up }}$} & \multicolumn{2}{|c|}{ Exerciseindividual exercises type } & \multirow{2}{*}{$\frac{\text { Intensity (RPE) }}{11-13}$} & \multirow{2}{*}{$\begin{array}{l}\text { Time }(\min ) \\
5\end{array}$} \\
\hline & Stretching & & & \\
\hline \multirow[t]{2}{*}{ Main exercise } & Aerobic & $\begin{array}{l}\text { Walking in place } \\
\text { Simple gymnastics }\end{array}$ & $12-16$ & $10-30$ \\
\hline & $\begin{array}{l}\text { Resistance (with resist- } \\
\text { ance bands) }\end{array}$ & $\begin{array}{l}\text { Seated chair leg flexion } \\
\text { Seated chair hip joint rotation } \\
\text { Standing hip extension } \\
\text { Standing hip flexion } \\
\text { Standing knee flexion } \\
\text { Standing knee extension } \\
\text { Wall squat }\end{array}$ & $11-15$ & \\
\hline Cool-down & Stretching & & $11-13$ & 5 \\
\hline
\end{tabular}

$R P E$ rating of perceived exertion

Fig. 3 Set-up for the smartphone mirroring-based telepresence exercise program used in this randomized controlled trial in obese older South Korean women

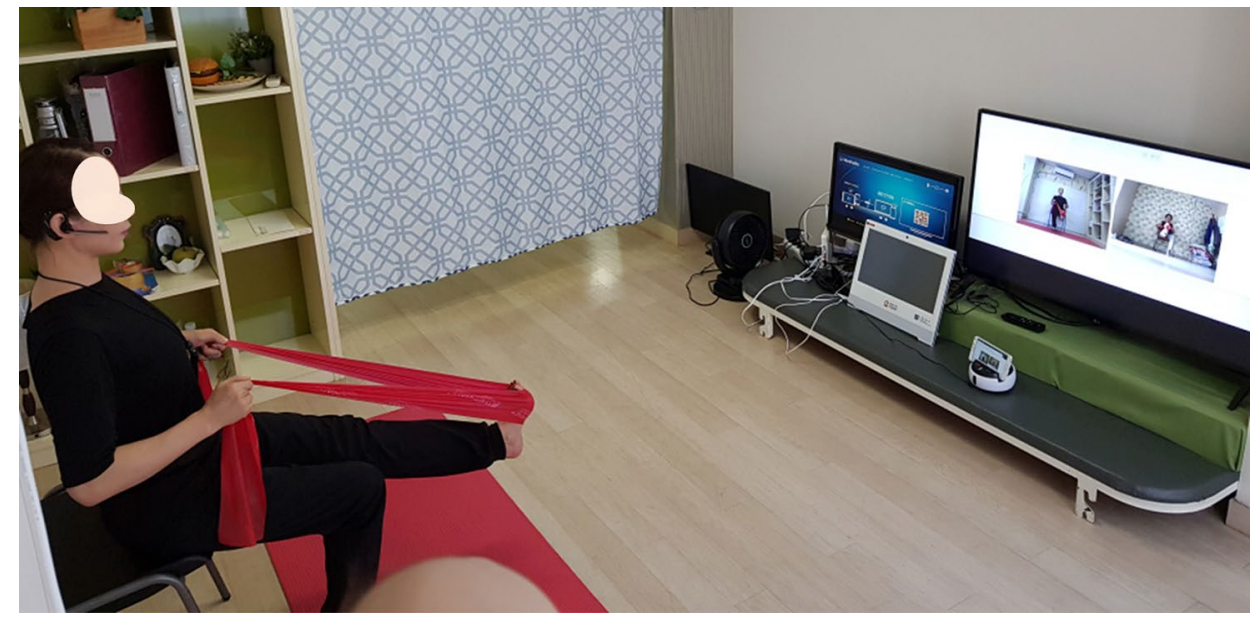




\section{Control group}

Nineteen participants in the control group performed the group exercise programs, which was conducted by an instructor at the senior citizen center, similar to the intervention group. Except for the place, all guidelines were the same as those for the intervention group.

\section{General procedures}

Each participant in both groups received an explanation and instructions for the exercise program. Once a month during the program, all participants were provided with nutrition advice and fitness education.

During the education sessions, the researchers checked whether the participants were maintaining their usual physical activity and food intake. The intervention lasted for 20-40 min, three times a week for 12 weeks.

\section{Outcome measures}

Body composition and physical function were examined before and after the intervention by an exercise specialist.

\section{Body composition}

Body composition was evaluated based on the percentage of body fat and skeletal muscle mass, measured using bioelectrical impedance analysis (InBody S10; InBody Co., Ltd., Seoul, South Korea). After the height and weight had been measured, four electrodes were attached to the upper and lower extremities in the supine position.

\section{Physical function}

The Short Physical Performance Battery (SPPB) and handgrip strength (HGS) were used to evaluate physical functional ability $[23,24]$. The SPPB was performed according to the standard procedure described in the literature [25]. Participants were asked to perform three tests: standing balance, a 4-m walk at their usual pace, and standing up five times from a seated position. HGS was expressed in $\mathrm{kg}$ and measured using a hydraulic hand dynamometer (Takei Dynamometer Model T.K.K. 5401, Takei Scientific Instruments Co., Ltd., Niigata City, Japan) that had been calibrated with a weight of $2 \mathrm{~kg}$ [26]. The measurement was repeated twice with each hand, with a recovery interval of 1 min between each measurement.
Body composition, SPPB, and grip strength were assessed at baseline (pre-test) and at the end of the 12-week study period (post-test).

\section{Statistical analyses}

\section{Sample size calculation}

A power analysis was performed using $\mathrm{G}^{*}$ Power 3 [27] and revealed that a sample size of 12 and 17 participants for the IG and CG, respectively, was sufficient to distinguish an effect size of 0.30 with an $\alpha$-value (probability of type 1 error) of 0.05 and a statistical power $(1-\beta)$ of 0.80 . All measured variables were presented as mean \pm standard deviation. An independent $t$ test was used to test the homogeneity of physical characteristics in the participants between the two groups before the intervention. A two-way repeatedmeasures analysis of variance (ANOVA) was performed to account for the effect of group (IG and CG), time (preand post-test), and the interaction between group and time. When significant interaction effects were observed, a paired $t$ test was used to assess within-group differences between pre- and post-test measurements. The statistical significance threshold was $P<0.05$ for all tests. All statistical analyses were performed using IBM SPSS Statistics for Windows version 22.0 (IBM Corp., Armonk, NY, USA).

\section{Results}

\section{General characteristics of the participants}

The homogeneity test for physical characteristics, physical function, and body composition showed that at baseline, the IG and CG did not differ significantly in terms of participants' age, body weight, height, physical function, and body fat percentage (Table 2).

Table 2 Baseline physical characteristics of obese older women $(n=29)$ undergoing an exercise program

\begin{tabular}{lrrr}
\hline & $\begin{array}{l}\text { Interven- } \\
\text { tion group } \\
(n=12)\end{array}$ & $\begin{array}{l}\text { Control group } \\
(n=17)\end{array}$ & $P$ value \\
\hline Age (years) & $78.41 \pm 2.84$ & $81.05 \pm 3.96$ & 0.06 \\
Weight $(\mathrm{kg})$ & $60.10 \pm 7.56$ & $57.15 \pm 8.78$ & 0.35 \\
Height $(\mathrm{cm})$ & $149.20 \pm 5.13$ & $148.84 \pm 4.97$ & 0.85 \\
Percent body fat $(\%)$ & $41.13 \pm 7.14$ & $41.87 \pm 5.84$ & 0.76 \\
\hline
\end{tabular}

Values are presented as mean \pm standard deviation of physical measurements. $P$ values represent homogeneity and were obtained using an independent $t$ test 
Table 3 Changes in body composition in obese older women $(n=29)$ undergoing a smartphone mirroring-based telepresence exercise program or group exercises

\begin{tabular}{llllll}
\hline & Group & Pre-test & Post-test & & $P$ value \\
\hline Weight (kg) & IG & $60.10 \pm 7.56$ & $59.47 \pm 6.69$ & Time & 0.004 \\
& CG & $57.15 \pm 8.78$ & $56.36 \pm 8.96^{\dagger}$ & Group & 0.336 \\
& & & & Time $\times$ group & 0.713 \\
Percent body fat (\%) & IG & $41.13 \pm 7.14$ & $39.76 \pm 7.29^{*}$ & Time & 0.000 \\
& CG & $41.87 \pm 5.84$ & $40.32 \pm 6.03^{\dagger}$ & Group & 0.791 \\
& & & & Time $\times$ group & 0.771 \\
Skeletal muscle mass (kg) & IG & $18.45 \pm 1.72$ & $18.73 \pm 1.45$ & Time & 0.029 \\
& CG & $16.91 \pm 2.07$ & $17.09 \pm 2.10^{*}$ & Group & 0.034 \\
& & & & Time $\times$ group & 0.645 \\
\hline
\end{tabular}

Values are presented as mean \pm standard deviation. $P$ values represent two-way repeated-measures ANOVA $I G$ intervention group, $C G$ control group

${ }^{*} P<0.05,{ }^{\dagger} P<0.01$

\section{Body composition}

Table 3 shows changes in body composition between baseline and after the 12-week intervention period. Two-way repeated-measures ANOVA revealed no significant interaction effects on all variables. The measurement time showed a significant effect on weight $(P=0.004)$ and body fat percentage $(P<0.001)$. With regard to skeletal muscle mass, the measurement time $(P=0.029)$ and group $(P=0.034)$ had significant effects. In the comparison of the values before with those after the intervention, weight $(P=0.006)$ significantly decreased in the $\mathrm{CG}$, the body fat percentage decreased significantly in the IG $(P=0.026)$ and the $\mathrm{CG}$ $(P=0.001)$, and skeletal muscle mass $(P=0.44)$ significantly increased in the $\mathrm{CG}$.

\section{Physical function}

Table 4 shows changes in the SPPB and HGS from baseline to after the intervention. Two-way repeated-measures ANOVA revealed no significant interaction effects on all variables. The measurement time had no significant effect on the SPPB or right-hand grip strength, although it had a significant effect on the left-hand grip strength $(P=0.002)$. Both groups showed significant effects on the SPPB $(P<0.001)$, right grip $(P=0.039)$, and left grip $(P=0.042)$. The right grip $(P=0.000)$ significantly increased in the $\mathrm{CG}$, and the left grip $(P=0.000)$ significantly increased in the IG from before to after the intervention.

\section{Discussion}

In this study, we developed a smartphone mirroring-based telepresence exercise program capable of real-time personal feedback, tested it in 12 obese older women over 12 weeks, and analyzed the effect on body composition and physical function. The findings were compared with 17 women participating in an exercise group for the same period and showed a significant decrease in percentage body fat in both groups.

Increased body fat in older people causes an increase in tumor necrosis factor-alpha, interleukin-6, and monocyte chemoattractant protein-1 levels in fat tissue and a decrease
Table 4 Changes in the short physical performance battery score and grip strength in obese older women $(n=29)$ undergoing a smartphone mirroring-based telepresence exercise program or group exercises

\begin{tabular}{llllll}
\hline & Group & Pre-test & Post-test & & $P$ value \\
\hline SPPB (score) & IG & $8.25 \pm 2.26$ & $8.91 \pm 1.78$ & Time & 0.244 \\
& CG & $4.88 \pm 2.61$ & $5.47 \pm 2.87$ & Group & 0.000 \\
& & & & Time $\times$ group & 0.941 \\
Right grip (kg) & IG & $17.37 \pm 3.23$ & $27.62 \pm 27.46$ & Time & 0.075 \\
& CG & $13.91 \pm 4.07$ & $16.47 \pm 3.06^{* *}$ & Group & 0.039 \\
& & & & Time $\times$ group & 0.276 \\
Left grip (kg) & IG & $15.79 \pm 4.31$ & $18.08 \pm 2.42^{* *}$ & Time & 0.002 \\
& CG & $13.50 \pm 4.30$ & $15.11 \pm 2.93$ & Group & 0.042 \\
& & & & Time $\times$ group & 0.563 \\
\hline
\end{tabular}

Values are presented as mean \pm standard deviation. $P$ values represent a two-way repeated-measures analysis of variance

$S P P B$ Short Physical Performance Battery, $I G$ intervention group, $C G$ control group

$* P<0.05, * * P<0.01$ 
in adiponectin levels, which may lead to cardiovascular disease [28].

Although many traditional group exercises are delivered for weight control in obese older people, participation may be restricted if a person's home is far from the exercise location, or they have poor balance. The attendance rate of classes may also vary depending on the season and weather [12].

During the COVID-19 pandemic, the social distancing measures that encouraged older people to stay at home with limited daily activities may have unintentionally aggravated sedentary lifestyles and the incidence of obesity, type 2 diabetes, cancer [29], cardiovascular disease, and mortality [2]. During a lockdown, the only solution to stay active and exercise is to increase physical activity at home.

South Korea is an information technology powerhouse. Therefore, it is relatively easy for the elderly population to access fitness-related apps through their smartphones. However, these apps are of limited use to them because they cannot provide real-time feedback to users and do not consider the physical and cognitive characteristics of the elderly.

Previous studies have shown that indoor group exercise classes can decrease the body fat percentage in obese older people. To the best of our knowledge, this was the first study to compare a smartphone mirroring-based telepresence exercise program with indoor group exercises [30,31]. The smartphone exercise program was as effective as in-person group exercises in reducing body fat in obese older women.

Although the exercise time suggested in this study was slightly less than the 30-60 min daily exercise time recommended by the American College of Sports Medicine, the body fat percentage decreased in both groups. This is thought to be a result of the "Principle of Initial Values" [22], which implies that individuals with lower initial values respond faster to exercise than those with higher initial values, as their body fat percentage is higher than that of other research participants. As a result, weight loss and muscle mass increase were found in the CG. This result suggests that the movement of the women in the CG to the facility for the group exercise classes for 12 weeks may have had some influence on their energy expenditure. Consequently, to derive more accurate results, future research should use an activity tracker to control the total amount of activity in the participants [32].

A lack of physical activity accelerates the symptoms of muscle atrophy and loss with age and has a significant influence on the overall deterioration of function and movement required for an independent daily life. Furthermore, decreased balance control and walking ability with aging increase the risk of falling. The physical decline caused by aging largely results from diseases and poor lifestyle habits. If appropriate interventions such as exercise are implemented, some negative outcomes, such as falls, can be prevented [33]. For the elderly, physical strength is the most important element enabling them to perform their activities of daily life. Balance and lower body control are necessary for quick movements, for example, while getting on and off the bus, entering and exiting the bathtub, and doing kitchen work [34].

In this study, the SPPB and grip strength were measured to gauge balance and upper body strength, respectively. As a result of the intervention, the SPPB score increased, albeit not significantly, in both groups, and grip strength increased significantly in both groups. These results contrast those of a previous study that reported an improvement in balance through exercise in obese older women $[35,36]$. Compared with these studies, less time was dedicated to lower extremity strength training in the exercise program in this study. The ratio of lower extremity to upper extremity exercise time should be increased when applying the program to obese older women in the future.

Grip strength is a critical indicator of functional and cognitive ability and mobility that can also predict mortality. An improvement in grip strength is not simply related to muscle strength but to positive changes in cognitive ability, including mental and psychological factors, mobility, and independence [37, 38].

The intervention in this study increased grip strength in both exercise groups, which is believed to have resulted from the continuous stimulation of the hand muscles when holding the resistance band during the resistance program.

In this study, we explored the additional benefits and improvements of a smartphone-based telepresence exercise compared with conventional group exercises when addressing obesity in the elderly. First, the exercise instructor was able to provide immediate feedback after observing the participants' physical and cognitive capacity and correct any problems directly. This is very helpful for elderly people whose cognitive ability might not allow them to focus on the correct and precise movement execution during exercise. Second, participants could control the volume of the speaker's voice individually on their smartphones. This is an advantage because the hearing ability of the elderly may be impaired.

The results of this study should be interpreted within its limitations. First, the results cannot be generalized because of the small sample size. In future studies, more participants should be included. Second, during the evaluation, the researchers did not analyze the user-friendliness of this program. In future research, the program should be revised to evaluate the user-friendliness of the equipment and the smartphone-based telepresence exercise program as a tool. It should also be tested in various populations, such as those with disabilities and hospital patients. 


\section{Conclusions}

A smartphone-based telepresence exercise program was similarly effective in reducing the body fat percentage and grip strength as conventional group exercises in obese older women. This tool can be safely used by the elderly at home during the coronavirus pandemic.

Supplementary Information The online version contains supplementary material available at https://doi.org/10.1007/s40520-021-02033-4.

Author contributions Study concept and design: HJK and SSK; acquisition of data: JYH and HJJ; analysis and interpretation of data: JYH; drafting of the manuscript: all authors; critical revision of the manuscript: all authors; statistical analysis: JYH; obtained funding: JYH; administrative, technical, or material support: HJJ; and study supervision: HJK. All authors read and approved the final manuscript.

Funding This work was supported by the Ministry of Education of the Republic of Korea, National Research Foundation of Korea (NRF2019S1A5A8033341), and 2018 Moonsuk Research Grant from the Korean Society of Obesity.

Availability of data and materials Supplementary material included data and materials.

\section{Declarations}

Conflict of interest The authors declare no conflict of interest.

Statement of human and animal rights All procedures of the study have been conducted according to the principles expressed in the Declaration of Helsinki 1964 and its later amendments. This study was approved by the Seoul National University Hospital Institutional Review Board (approval no. 1804-060-936) and was registered with the Clinical Research Information Service, which is a primary registry of the World Health Organization International Clinical Trials Registry Platform.

Informed consent Informed consent was obtained from all individual participants included in the study.

Consent to participate All participants provided written informed consent before enrollment and were free to withdraw from the study at any time.

Consent for publication All authors explicitly consent for publication.

\section{References}

1. Statistics Korea (2019) Population aged 65 years and above in South Korea in 2019. Daejeon, South Korea

2. Hughes VA, Roubenoff R, Wood M et al (2004) Anthropometric assessment of 10-y changes in body composition in the elderly. Am J Clin Nutr 80:475-482. https://doi.org/10.1093/ajcn/80.2.475

3. Zamboni M, Mazzali G, Fantin F et al (2008) Sarcopenic obesity: a new category of obesity in the elderly. Nutr Metab Cardiovasc Dis 18:388-395. https://doi.org/10.1016/j.numecd.2007.10.002
4. Sarma S, Sockalingam S, Dash S (2021) Obesity as a multisystem disease: trends in obesity rates and obesity-related complications. Diabetes Obes Metab 23:3-16. https://doi.org/10.1111/dom.14290

5. Piché ME, Tchernof A, Després JP (2020) Obesity phenotypes, diabetes, and cardiovascular diseases. Circ Res 126:1477-1500. https://doi.org/10.1161/CIRCRESAHA.120.316101

6. Orsini N, Bellocco R, Bottai M et al (2006) Age and temporal trends of total physical activity among Swedish women. Med Sci Sports Exerc 38:240-245. https://doi.org/10.1249/01.mss.00001 85086.19220.b3

7. Rolland Y, Lauwers-Cances V, Cristini C et al (2009) Difficulties with physical function associated with obesity, sarcopenia, and sarcopenic-obesity in community dwelling elderly women: the EPIDOS (EPIDemiologie de l'OSteoporose) Study. Am J Clin Nutr 89:1895-1900. https://doi.org/10.3945/ajen.2008.26950

8. Fülöp T Jr, Wórum I, Csongor J et al (1985) Body composition in elderly people. I. Determination of body composition by multiisotope method and the elimination kinetics of these isotopes in healthy elderly subjects. Gerontology 31:6-14. https://doi.org/10. $1159 / 000212676$

9. Schilke JM (1991) Slowing the aging process with physical activity. J Gerontol Nurs 17:4-8. https://doi.org/10.3928/0098-913419910601-04

10. Kong SY, Kim SS (2017) Elastic band exercise's and aerobic exercise's influences on the elderly people's health-relevant basic fitness and vascular health. Int J Hum Mov Sports Sci 56:703-711

11. Lee BS, Kim KA, Lee MG (2017) Effects of 10 weeks of aerobic exercise training on cardiovascular function, atherosclerosis, and vascular endothelial function in elderly women. Korean J Sport Sci 28:300-314

12. Johnston JD, Massey AP, Devaneaux CA (2012) Innovation in weight loss programs: a 3-dimensional virtual-world approach. J Med Internet Res 14:e120. https://doi.org/10.2196/jmir.2254

13. Schuch F, Bulzing R, Meyer J et al (2021) Moderate to vigorous physical activity and sedentary behavior change in self-isolating adults during the COVID-19 pandemic in Brazil: a cross-sectional survey exploring correlates. Sport Sci Health. https://doi.org/10. 1007/s11332-021-00788-x

14. Cunningham C, Osullivan R, Caserotti P et al (2020) Consequences of physical inactivity in older adults: a systematic review of reviews and meta-analyses. Scand J Med Sci Sports 30:816827. https://doi.org/10.1111/sms.13616

15. Riley WT, Rivera DE, Atienza AA et al (2011) Health behavior models in the age of mobile interventions: are our theories up to the task? Transl Behav Med 1:53-71. https://doi.org/10.1007/ s13142-011-0021-7

16. Payne HE, Lister C, West JH et al (2015) Behavioral functionality of mobile apps in health interventions: a systematic review of the literature. JMIR MHealth UHealth 3:e20. https://doi.org/10.2196/ mhealth.3335

17. Patrick K, Griswold WG, Raab F et al (2008) Health and the mobile phone. Am J Prev Med 35:177-181. https://doi.org/10. 1016/j.amepre.2008.05.001

18. Hong J, Kong HJ, Yoon HJ (2018) Web-based telepresence exercise program for community-dwelling elderly women with a high risk of falling: randomized controlled trial. JMIR Mhealth Uhealth 6:e132. https://doi.org/10.2196/mhealth.9563

19. American College of Sports Medicine, Riebe D, Ehrman JK et al (2018) ACSM's guidelines for exercise testing and prescription. Wolters Kluwer, Philadelphia

20. Korean Society of Obesity, Korean Society of Exercise Physiology (2016) Exercise manual for obesity management. Chungwoon University, Seoul, p 25

21. Borg GA (1982) Psychophysical bases of perceived exertion. Med Sci Sports Exerc 14:377-381 
22. Heyward VH (2010) Advanced fitness assessment and exercise prescription. In: Human kinetics, 6th edn. Champaign

23. Guralnik JM, Simonsick EM, Ferrucci L et al (1994) A short physical performance battery assessing lower extremity function: association with self-reported disability and prediction of mortality and nursing home admission. J Gerontol 49:M85-M94. https:// doi.org/10.1093/geronj/49.2.m85

24. Amaral CA, Amaral TLM, Monteiro GTR et al (2019) Hand grip strength: reference values for adults and elderly people of Rio Branco, Acre, Brazil. PLoS ONE 14:e0211452. https://doi.org/ 10.1371/journal.pone.0211452

25. Lauretani F, Ticinesi A, Gionti L et al (2019) Short-physical performance battery (SPPB) score is associated with falls in older outpatients. Aging Clin Exp Res 31:1435-1442. https://doi.org/ 10.1007/s40520-018-1082-y

26. Fess EE (2002) Documentation: essential elements of an upper extremity assessment battery. In: Mackin EJ, Callahan AD, Skirven TM et al (eds) Rehabilitation of the hand and upper extremity, 5th edn. Mosby, St Louis, pp 263-284

27. Faul F, Erdfelder E, Lang AG et al (2007) G*Power 3: a flexible statistical power analysis program for the social, behavioral, and biomedical sciences. Behav Res Methods 39:175-191. https://doi. org/10.3758/bf03193146

28. Zamboni M, Zoico E, Scartezzini T et al (2003) Body composition changes in stable-weight elderly subjects: the effect of sex. Aging Clin Exp Res 15:321-327. https://doi.org/10.1007/BF03324517

29. Patterson R, McNamara E, Tainio M et al (2018) Sedentary behaviour and risk of all-cause, cardiovascular and cancer mortality, and incident type 2 diabetes: a systematic review and dose response meta-analysis. Eur J Epidemiol 33:811-829. https://doi.org/10. 1007/s10654-018-0380-1

30. Kemmler W, von Stengel S, Engelke K et al (2010) Exercise, body composition, and functional ability: a randomized controlled trial. Am J Prev Med 38:279-287. https://doi.org/10.1016/j.amepre. 2009.10.042
31. Park SK, Kim EH, Kwon YC et al (2010) Effects of combined exercise program on health-related physical fitness, anti-aging hormone and prevention of sarcopenia in elderly women with sarcopenia. J Sport Leis Stud 40:435-442

32. Arnett SW, Laity JH, Agrawal SK et al (2008) Aerobic reserve and physical functional performance in older adults. Age Ageing 37:384-389. https://doi.org/10.1093/ageing/afn022

33. Hyeon IS, Park M, Park KM et al (2010) The effects of a fall prevention program on the low-income elderly at risk of falls. J Korean Acad Community Health Nurs 21:200-209. https://doi. org/10.12799/jkachn.2010.21.2.200

34. Rikli RE, Jones CJ (2001) Senior fitness test manual. Human Kinetics, Champaign

35. Villareal DT, Banks M, Sinacore DR et al (2006) Effect of weight loss and exercise on frailty in obese older adults. Arch Intern Med 166:860-866. https://doi.org/10.1001/archinte.166.8.860

36. Anton SD, Manini TM, Milsom VA et al (2011) Effects of a weight loss plus exercise program on physical function in overweight, older women: a randomized controlled trial. Clin Interv Aging 6:141-149. https://doi.org/10.2147/CIA.S17001

37. Rijk JM, Roos PR, Deckx L et al (2016) Prognostic value of handgrip strength in people aged 60 years and older: a systematic review and meta-analysis. Geriatr Gerontol Int 16:5-20. https:// doi.org/10.1111/ggi.12508

38. Stamatakis E, Gale J, Bauman A et al (2019) Sitting time, physical activity, and risk of mortality in adults. J Am Coll Cardiol 73:2062-2072. https://doi.org/10.1016/j.jacc.2019.02.031

Publisher's Note Springer Nature remains neutral with regard to jurisdictional claims in published maps and institutional affiliations. 